\title{
Quantum walks in weak electric fields and Bloch oscillations
}

\author{
Pablo Arnault $\odot,{ }^{1,2, *}$ Benjamin Pepper, ${ }^{1,3}$ and A. Pérez ${ }^{1, \dagger}$ \\ ${ }^{1}$ Departamento de Física Teórica and IFIC, Universidad de Valencia, CSIC, Cerrer del Dr. Moliner 50, 46100 Burjassot, Spain \\ ${ }^{2}$ Institute for Quantum Computing, 200 University Ave W, Waterloo, Ontario, Canada N2L 3G1 \\ ${ }^{3}$ Blackett Laboratory, Department of Physics, Imperial College London, Prince Consort Road, London SW7 2AZ, United Kingdom
}

(Received 23 March 2020; accepted 22 May 2020; published 19 June 2020)

\begin{abstract}
Bloch oscillations appear when an electric field is superimposed on a quantum particle that evolves on a lattice with a tight-binding Hamiltonian (TBH), i.e., evolves via what we call an electric TBH; this phenomenon will be referred to as TBH Bloch oscillations. A similar phenomenon is known to show up in so-called electric discrete-time quantum walks (DQWs) [C. Cedzich et al., Phys. Rev. Lett. 111, 160601 (2013); this phenomenon will be referred to as DQW Bloch oscillations. This similarity is particularly salient when the electric field of the DQW is weak. For a wide, i.e., spatially extended, initial condition, one numerically observes semiclassical oscillations, i.e., oscillations of a localized particle, for both the electric TBH and the electric DQW. More precisely, the numerical simulations strongly suggest that the semiclassical DQW Bloch oscillations correspond to two counterpropagating semiclassical TBH Bloch oscillations. In this work it is shown that, under certain assumptions, the solution of the electric DQW for a weak electric field and a wide initial condition is well approximated by the superposition of two continuous-time expressions, which are counterpropagating solutions of an electric TBH whose hopping amplitude is the cosine of the arbitrary coin-operator mixing angle. In contrast, if one wishes the continuous-time approximation to hold for spatially localized initial conditions, one needs at least the DQW to be lazy, as suggested by numerical simulations and by the fact that this has been proven in the case of a vanishing electric field [F. W. Strauch, Phys. Rev. A 74, 030301(R) (2006)].
\end{abstract}

DOI: 10.1103/PhysRevA.101.062324

\section{INTRODUCTION}

Quantum walks can be understood as quantum analogs of classical random walks. In the present work we focus on their discrete-time version, namely, discrete-time quantum walks (DQWs); these have become very popular in recent years since they were found to have numerous applications. One of their most relevant applications is quantum algorithmics[1,2]; see Ref. [3] for a compact historical review with references to key works in this field. Their other most relevant application, to which the present work is dedicated, is the quantum simulation of physical equations and phenomena. Indeed, DQWs can, most notably, quantum simulate the Dirac equation and, more generally, a quantum particle on a lattice in various regimes, subject to external electric and magnetic fields [4-11] and/or to an external relativistic gravitational field $[6,12-14]$. A variety of high-energy-physics phenomena and situations such as neutrino oscillations [15] and the presence of extra dimensions [16] have also been reproduced with DQWs.

In the present work we focus on the so-called electric DQWs on a one-dimensional (1D) lattice $[3,4,6]$. Notice that such an electric DQW has been implemented experimentally with cold atoms in an optical lattice (see Ref. [8]). These electric DQWs we deal with are called electric for the following reasons. First, such an electric DQW is a possible discretiza-

\footnotetext{
*pablo.arnault@ific.uv.es

†armando.perez@uv.es
}

tion of the Dirac equation in an external electric field; in other words, such an electric DQW reproduces, in the continuum limit, the Dirac equation in an external electric field $[3,6]$. Second, these electric DQWs present an exact lattice gauge invariance, in the fashion of lattice gauge theories $[3,6,10]$; in the continuum limit, this lattice gauge invariance tends to the usual electromagnetic gauge invariance of the Dirac equation. The fact that the typical phenomenology exhibited by these electric DQWs, that is, as we will review, Blochlike oscillations, share close similarities with usual solid-state Bloch oscillations is due to the presence of the lattice (which disappears in the continuum); the analysis of these similarities is the point of our work. In relation to the lattice gauge invariance mentioned above, and because we work (i.e., define our evolution scheme) at the level of the evolution operator for the walker [which is similar to working at the level of the (effective) Hamiltonian] and not at the level of the forces, there is not just one but a family of electric potentials (that is, phases that we can make the walker catch at each time step), which will yield the same electric field and hence the same evolution of the probability distribution. One possibility for an external constant and uniform electric field is to implement, at each time step, an extra, position-dependent phase. This is what we do in our work and what was done in Ref. [8]. In such experimental realizations with quantum machines, one speaks of an artificial or synthetic electric field, because it is not an actual external electric field which is applied to make the walker catch this extra phase; what is done, for example, in Ref. [8] is to accelerate the optical lattice in which the walker (a cesium atom) is trapped. 
As mentioned in the preceding paragraph, one of the observed features in electric DQWs is the appearance of oscillations having the usual Bloch period, inversely proportional to the electric field, which we will refer to as DQW Bloch oscillations $[4,10,17,18]$. Bloch oscillations can also be observed when a similar DQW is implemented in quasimomentum space, rather than in position space [19]. Note also that DQW Bloch oscillations have been proposed as a means for the direct measurement of topological invariants [20].

The standard well-known Bloch oscillations appear when an electric field is superimposed on a quantum particle that evolves on a lattice with a tight-binding Hamiltonian (TBH) [21-24]; we will speak of electric TBHs and TBH Bloch oscillations. Even if DQW Bloch oscillations have already been discussed in the literature, their relationship to TBH Bloch oscillations has not been analyzed in detail. More precisely, we wish here to investigate the case where the oscillations appear in a semiclassical manner, i.e., show up as oscillations of a localized particle. For electric TBHs, it is known that an initial condition which superposes only a few lattice sites will produce not semiclassical oscillations, but so-called breathing modes and we will see that, in this situation, similar breathing modes appear also in the electric DQW when the electric field is chosen to be weak, i.e., small with respect to its maximum value $\pi$. In order to obtain semiclassical oscillations in the electric TBH, one needs a combination of many sites, such as a wide Gaussian state [21]. The question that arises is whether the same requirement holds for the DQW: One can readily see numerically that it does and that these semiclassical DQW Bloch oscillations seem to correspond to the superposition of two counterpropagating semiclassical TBH Bloch oscillations.

The main achievement of the present article would be to provide an analytical expression supporting these numerical observations. As we will show, if both the electric field is chosen to be weak and the initial condition is wide, i.e., large with respect to the lattice spacing, one can in the end approximate the probability distribution of the electric DQW with a continuous-time analytical expression. This expression is, both in quasimomentum and in real space, the superposition of two counterpropagating solutions of an electric TBH whose hopping amplitude is the cosine of the arbitrary coin-operator mixing angle. The continuous-time differential equation we derive along the way under certain assumptions can be written in terms of a certain Hamiltonian, which takes the form of the free part of the electric TBH just mentioned. The possibility of establishing such a simple connection between the electric DQW and the electric TBH ultimately sheds light on the nature and behavior of DQW Bloch oscillations when the electric field is weak. The power of the result is that we do not require the free part of the electric DQW to be lazy as in Strauch's work [25], a situation which would be directly describable by a continuous-time quantum walk (CQW) whose Hamiltonian is simply an electric TBH. The description here is more involved, as we will see.

The article is organized as follows. In Sec. II we first introduce the electric DQW and some of its basic properties and then discuss the condition under which semiclassical TBH Bloch oscillations and DQW Bloch oscillations appear, that is, requiring the initial condition to be wide with respect to the lattice spacing. In Sec. III we define two-step dynamics from the evolution of the electric DQW and derive, when the electric field is weak, a continuous-time differential equation from it, which can be used to approximate the DQW evolution if the initial condition is wide. This continuous-time differential equation can be solved, which gives continuous-time formulas that for wide initial conditions are expected to be good approximations of (and can simply be compared with) the exact dynamics of the DQW, obtained by numerical simulation. Section IV is devoted to quantifying the agreement between the exact probability distribution and its continuoustime approximation. A summary and our main conclusions are presented in Sec. V. Certain enlightening or secondary calculations have been relegated to the Appendixes.

\section{ELECTRIC DISCRETE-TIME QUANTUM WALK ON THE LINE}

\section{A. Definition and basic properties}

The Hilbert space of the particle walking on the 1D lattice is $\mathcal{H}=\mathcal{H}_{\text {spatial }} \otimes \mathcal{H}_{\text {coin }}$, where $\mathcal{H}_{\text {spatial }}$ is the position Hilbert space, spanned by the lattice position states $\left\{\left|x_{n}=n a\right\rangle \mid n \in\right.$ $\mathbb{Z}$, with $a>0$ the lattice spacing, and where $\mathcal{H}_{\text {coin }}$ is the Hilbert space of an internal state of the walker which is called a coin, or chirality state (we discuss below why this term is used), that must be introduced. Indeed, the minimum dimension for $\mathcal{H}_{\text {coin }}$ is 2 if we want the evolution operator of the walker evolving on the space-time lattice to be local, unitary, and translationally invariant [26]. We work with this minimum dimension for $\mathcal{H}_{\text {coin }}$ and introduce a basis of the latter $\{|R\rangle,|L\rangle\}$, where $R$ and $L$ correspond to right and left, respectively (explained below). We will work with the identifications $|R\rangle=(1,0)^{\top}$ and $|L\rangle=(0,1)^{\top}$, where $\top$ denotes the transposition.

The state of the particle at the discrete time $j \in \mathbb{N}$ is described by the vector $\left|\Psi_{j}\right\rangle \in \mathcal{H}$ and is updated via

$$
\left|\Psi_{j+1}\right\rangle=W_{\phi}(\hat{x}, \hat{k})\left|\Psi_{j}\right\rangle,
$$

under the action of the unitary operator

$$
W_{\phi}(\hat{x}, \hat{k}) \equiv\left(e^{i \hat{x} \phi} \otimes I_{\text {coin }}\right) W_{0}(\hat{k})
$$

where

$$
W_{0}(\hat{k}) \equiv S(\hat{k})\left(\hat{I}_{\text {spatial }} \otimes C\right) .
$$

We use circumflexes for operators acting on the position Hilbert space, but not for those acting on the coin Hilbert space. The operators $\hat{x}$ and $\hat{k}$ are, respectively, the position and quasimomentum operators. The operator $e^{i \hat{x} \phi}$ implements, on the free ${ }^{1}$ walk operator $W_{0}(\hat{k})$, this special electric field $\phi \in \mathbb{R}$ that we have talked about in the Introduction, which is upper bounded by $2 \pi / a$ [4]. The free walk operator is the combination of (i) an operation acting solely on the coin state, implemented by the so-called coin operator $C$, which at this stage is an arbitrary $2 \times 2$ unitary matrix, and (ii) a

\footnotetext{
${ }^{1}$ The word "free" is used as usual in physics, i.e., it refers to translationally invariant dynamics.
} 
coin-dependent shift operator $S(\hat{k})$, which can be written, in the coin basis, as

$$
S(\hat{k}) \equiv e^{-i \sigma^{3} \hat{k} a}=\left[\begin{array}{cc}
e^{-i \hat{k} a} & 0 \\
0 & e^{i \hat{k} a}
\end{array}\right],
$$

where $\sigma^{3}=\operatorname{diag}(1,-1)$ is the third Pauli matrix. Note that $S(\hat{k})$ shifts by one lattice site to the right the up coin component of the state and the same but to the left for the down one, these coin components being, respectively,

$$
\begin{aligned}
\left|\psi_{j}^{R}\right\rangle & \equiv\left\langle R \mid \Psi_{j}\right\rangle, \\
\left|\psi_{j}^{L}\right\rangle & \equiv\left\langle L \mid \Psi_{j}\right\rangle .
\end{aligned}
$$

Finally, $I_{\text {coin }}=\mathbf{1}_{2}$ (the $2 \times 2$ identity matrix) and $\hat{I}_{\text {spatial }}$ are the identity operators acting on $\mathcal{H}_{\text {coin }}$ and $\mathcal{H}_{\text {spatial }}$, respectively, which we will often omit from now on to simplify the notation. In what follows we take the lattice spacing as the length unit $a=1$.

Let us examine the dynamics induced by $W_{\phi}(\hat{x}, \hat{k})$ in quasimomentum space, i.e., on the quasimomentum basis of $\mathcal{H}_{\text {spatial }}$, namely, $\{|k\rangle \mid k \in[-\pi, \pi[\}$, satisfying

$$
|k\rangle=\sum_{n} e^{i k x_{n}}\left|x_{n}\right\rangle .
$$

We make use of the fact that $e^{i \hat{x} \phi}$ induces translations in quasimomentum space, $e^{i \hat{x} \phi}|k\rangle=|k+\phi\rangle$, whereas $W_{0}(\hat{k})$ is diagonal in this basis, described by a $2 \times 2$ matrix $W_{0}(k)$. Acting with $\langle k|$ on the left-hand side of Eq. (1), we arrive at

$$
\tilde{\Psi}_{j+1}(k)=W_{0}(k-\phi) \tilde{\Psi}_{j}(k-\phi),
$$

where we have defined the two-component wave function

$$
\tilde{\Psi}_{j}(k) \equiv\left\langle k \mid \Psi_{j}\right\rangle .
$$

Equation (7) shows that the dynamics of the electric DQW defined in Eq. (2) can be described as the composition of two effects: the displacement in quasimomentum space, followed by the action of the free walk operator. This dynamics has been examined both in quasimomentum space and in the original position space and shows a rich behavior, depending on whether the ratio $\phi / 2 \pi$ is a rational or an irrational number $[4,5,7,27]$.

\section{B. Bloch oscillations}

\section{Breathing modes}

For a quantum particle with no internal degree of freedom moving on a lattice via a TBH, Bloch oscillations can be described as a consequence of the displacement in quasimomentum space due to the presence of an external constant and uniform force field, e.g., that induced by a constant and uniform electric field $E$, and they manifest as an oscillation of the probability distribution with a characteristic period $T_{\text {Bloch }}(E)=$ $2 \pi / E$, the so-called Bloch period, which is inversely proportional to the field $E$ (for a review, see Refs. [21,22]). A similar phenomenon has been observed in DQWs $[10,27]$, the Bloch period being in this case $T_{\text {Bloch }}(\phi)=2 \pi / \phi$. We give in the present section additional details in order to progressively develop the main result of this work.

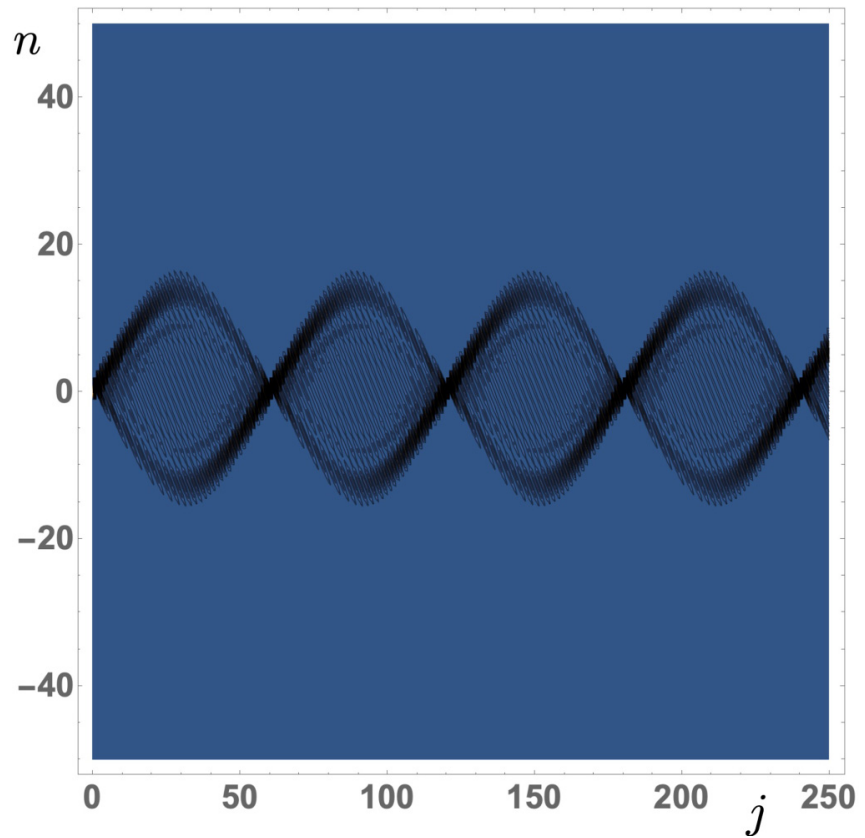

FIG. 1. Time evolution of the probability density $P_{j, n}$, where $n \in$ $\mathbb{Z}$ is the lattice site and $j \in \mathbb{N}$ the time instant. The initial state of the walker is a product state initially localized at the origin in position space and with coin state $\frac{1}{\sqrt{2}}(1,1)^{\top}$, although almost the same plot is reproduced with the coin state $\frac{1}{\sqrt{2}}(1,-1)^{\top}$ used in next plots. The coin operator is the Hadamard one, $\theta=\pi / 4$, and the electric field is $\phi=2 \pi / 60 \simeq 0.1$.

We choose, for the following numerical simulations, the coin operator to be

$$
C=\left(\begin{array}{cc}
\cos \theta & \sin \theta \\
\sin \theta & -\cos \theta
\end{array}\right) .
$$

Note that the extensively used Hadamard gate corresponds to $\theta=\pi / 4$. Numerical simulations of DQWs often use a state localized in position space as the initial condition. To see the effect of the electric field in this case, we run a numerical simulation of Eq. (1) with an initial state localized at the origin. Figure 1 shows the time evolution of the probability density $P_{j, n}$, defined by

$$
P_{j, n} \equiv\left\langle\Psi_{j} \mid x_{n}\right\rangle\left\langle x_{n} \mid \Psi_{j}\right\rangle,
$$

and, for the sake of completeness, we show in Fig. 2 the standard deviation $\sigma_{j}$, defined by

$$
\sigma_{j}=\sqrt{\left\langle n^{2}\right\rangle_{j}-\left(\langle n\rangle_{j}\right)^{2}},
$$

where

$$
\langle n\rangle_{j}=\sum_{n} n P_{j, n}
$$

and

$$
\left\langle n^{2}\right\rangle_{j}=\sum_{n} n^{2} P_{j, n}
$$

are, respectively, the average position and average squared position at time $j$. 


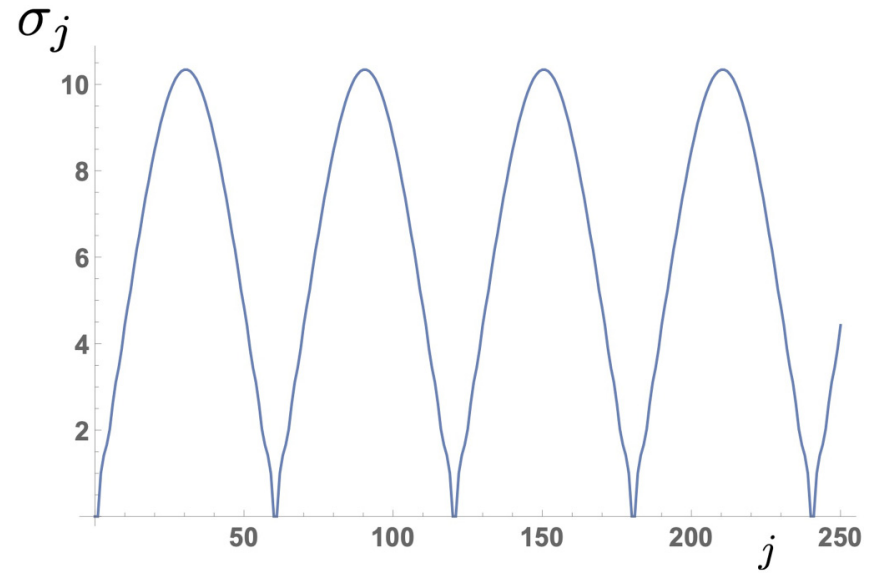

FIG. 2. Time evolution of the standard deviation $\sigma_{j}$, where $j \in$ $\mathbb{N}$ is the time instant, for the same conditions as in Fig. 1.

For the timescale we choose, we observe oscillations around the origin with a period given by the Bloch period $T_{\text {Bloch }}(\phi)$, as announced. These oscillations correspond to a splitting of the initial state into two components that tend to meet after a time $T_{\mathrm{Bloch}}(\phi)$. A similar phenomenon also appears with electric TBHs [21,22] when the initial state is localized in position space and is referred to as breathing modes.

Let us mention the differences between DQW Bloch oscillations and TBH Bloch oscillations. First of all, in DQWs, the recombination (usually called revival [4]) at the origin at multiples of $T_{\mathrm{Bloch}}(\phi)$ is not perfect; this translates in particular into a nonvanishing standard deviation (not visible in Fig. 2). More importantly, after some time, the walker eventually moves away from the origin ballistically, i.e., asymptotically one finds $\sigma_{j} \propto j$. These features, i.e., the revivals and then the ballistic excursion, actually occur only for rational values of $\phi / 2 \pi$ [4], while Anderson localization occurs for almost all irrational values [27]. For a discussion, we refer the reader to Refs. [4,5,7]. Regarding TBH Bloch oscillations, the level statistics of the corresponding model (Harper's equation) were studied in Ref. [28].

\section{Semiclassical Bloch oscillations in position space}

The situation described in Sec. II B 1 for both TBHs and DQWs is that observed for an initially localized walker. Now, in TBHs, the situation changes when one considers extended initial conditions, since in this case one obtains a wave packet which oscillates around the starting position, which is the semiclassical prediction. The question that arises is whether this kind of behavior has a parallelism in DQWs. To investigate this question, we first perform numerical simulations considering an initial product state with Gaussian spatial part

$$
\Psi_{0, n} \equiv\left\langle x_{n} \mid \Psi_{0}\right\rangle=c_{n}|s\rangle
$$

where $|s\rangle$ is the initial coin state and

$$
c_{n}=\frac{e^{-\beta n^{2}}}{\sqrt{\vartheta_{3}\left(0, e^{-2 \beta}\right)}}
$$

describes a Gaussian, which, because it is defined on a discrete space, must be normalized, in order to get $\sum_{n}\left|c_{n}\right|^{2}=1$, via

$$
\vartheta_{3}(u, q)=1+\sum_{n=1}^{\infty} q^{n^{2}} \cos (2 n u),
$$

the third Jacobi theta function.

For high values of $\phi$, i.e., of the order of $2 \pi$, there is a rich phenomenology. However, as $\phi$ is lowered, the contour plots of the probability distribution tend to converge towards a simple pattern, namely, oscillations of a localized particle with the Bloch period $T_{\mathrm{Bloch}}(\phi)$. This tendency is illustrated in Fig. 3, where we present $P_{j, n}$ for a strong and a weak $\phi$. As can be seen from the plots, the oscillations become smoother as $\phi$ decreases, and of course the Bloch period increases. Similar observations can be extracted from Fig. 4, by observing the curves that show the average position $\langle n\rangle_{j}$ which are obtained by lowering $\phi$.

An important message is obtained by observing Fig. 4(b), which plots the standard deviation $\sigma_{j}$ as a function of the time step $j$ for the same values $\phi=2 \pi / 20,2 \pi / 30$, and $2 \pi / 40$. Since $\phi / 2 \pi$ is a rational number in these plots, one should observe a long-term ballistic behavior with (imperfect) revivals, as proven in [4] for all kind of initial states (including the initial Gaussian states considered here). This effect is apparent for $\phi=2 \pi / 20$, but becomes less visible for lower values of $\phi$.

Figure 5 plots the minima of $\sigma_{j}$, as obtained from the curves represented in Fig. 4(b). As can be seen from this figure, this magnitude increases with $j$ (in this sense, the revivals become more imperfect as the time step increases). However, this tendency becomes quickly unobservable as $\phi$ is decreased for the same number of time steps. In fact, for $\phi=2 \pi / 60$ (not shown) the minima, during the same total duration, are constant within the machine precision.

These observations indicate a convergence of the dynamics for weak fields and encourage us to derive an analytical expression in the regime we are interested in: small $\phi$ and wide initial conditions. This is the goal of the next section.

\section{CONTINUOUS-TIME APPROXIMATION FOR WEAK ELECTRIC FIELDS AND WIDE INITIAL CONDITIONS}

\section{A. Introduction: The two branches arising from the two-step dynamics}

We start by rewriting Eq. (7) as

$$
\tilde{\Psi}_{j+1}(k+\phi)=W_{0}(k) \tilde{\Psi}_{j}(k) .
$$

Since $W_{0}(k)$ is unitary, one also has

$$
\tilde{\Psi}_{j-1}(k-\phi)=W_{0}^{\dagger}(k-\phi) \tilde{\Psi}_{j}(k),
$$

with $W_{0}^{\dagger}(k)$ the Hermitian conjugate of $W_{0}(k)$. By combining Eqs. (17) and (18) we arrive at

$$
\tilde{\Psi}_{j+1}(k+\phi)-\tilde{\Psi}_{j-1}(k-\phi)=\Delta_{\phi}(k) \tilde{\Psi}_{j}(k),
$$

where we have defined

$$
\Delta_{\phi}(k) \equiv W_{0}(k)-W_{0}^{\dagger}(k-\phi) .
$$

Equation (19) involves two time steps, from $j-1$ to $j+1$, so we call it two-step dynamics, in contrast to the original 

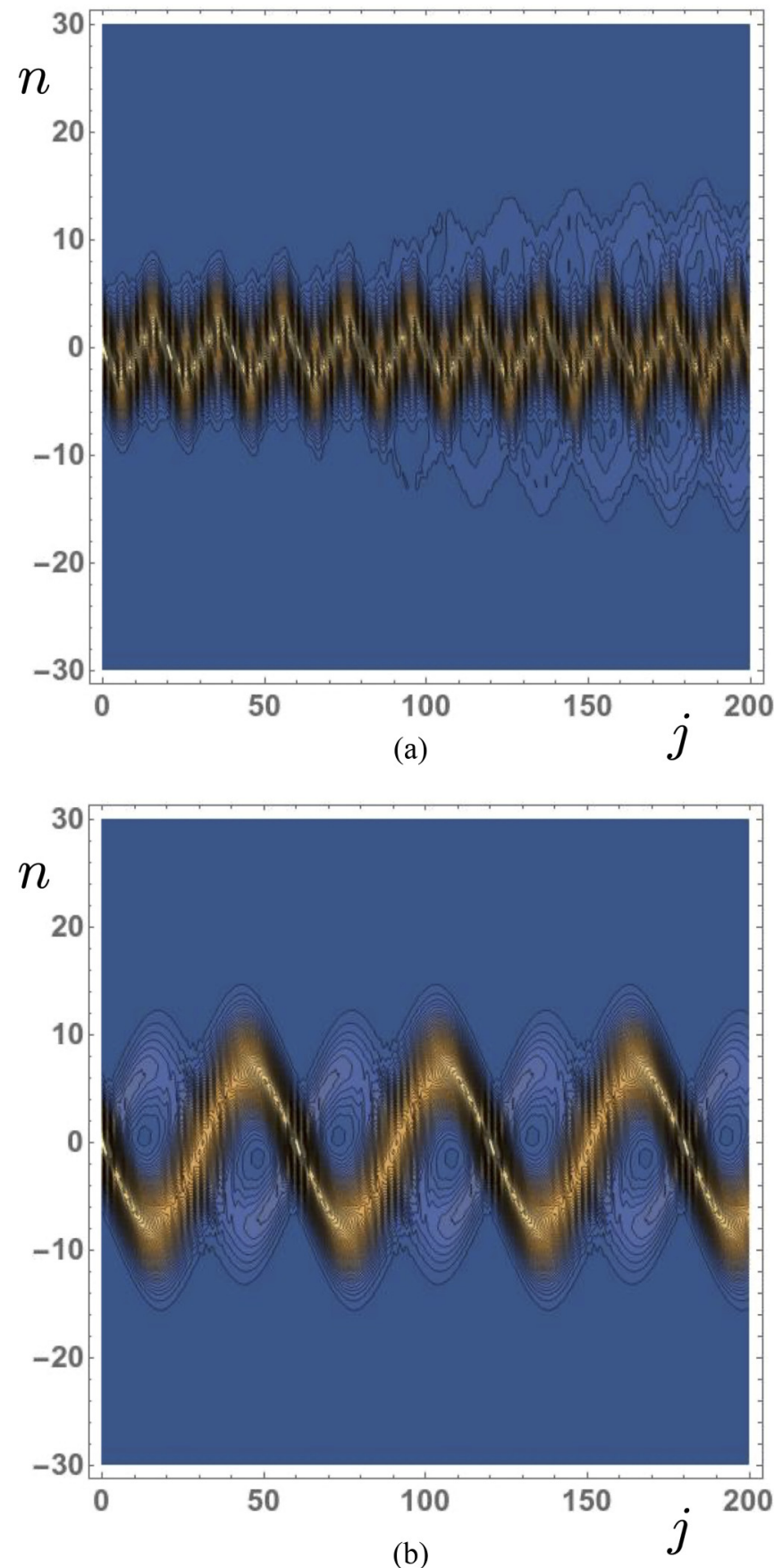

FIG. 3. Contour plots corresponding to the probability density $P_{j, n}$, as a function of the lattice site $n$ and the time instant $j$, obtained with (a) $\phi=2 \pi / 20$ and (b) $\phi=2 \pi / 60$. In the simulations, $\beta=$ 0.05 [see Eq. (15)]. The initial coin state is $|s\rangle=\frac{1}{\sqrt{2}}(1,-1)$.

dynamics (1), which involves a single time step, which we call one-step dynamics. The two-step dynamics takes as input not one but two initial conditions. It is easy to show by induction that the two-step and the one-step dynamics are equivalent provided we choose, for the two-step dynamics, the second initial condition given by the one-step dynamics,

$$
\left|\Psi_{1}\right\rangle=W_{0}(\hat{k})\left|\Psi_{0}\right\rangle,
$$

which we will assume in what follows.

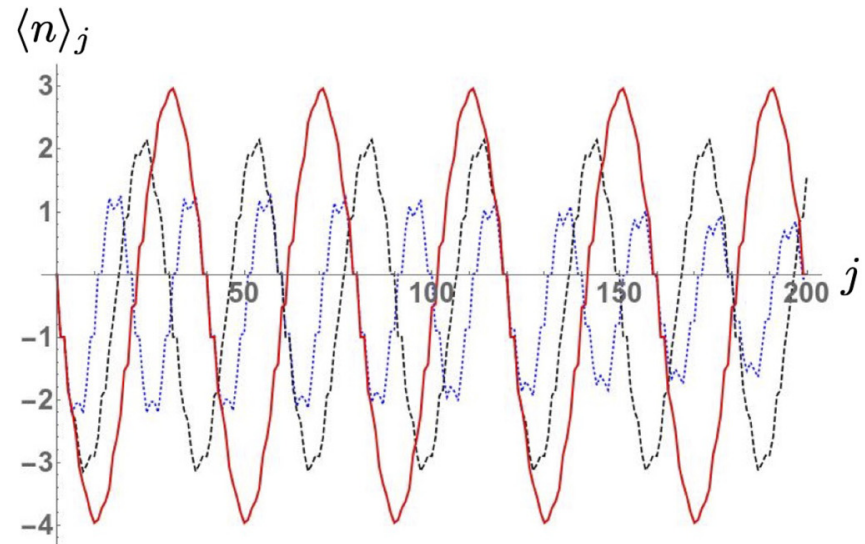

(a)

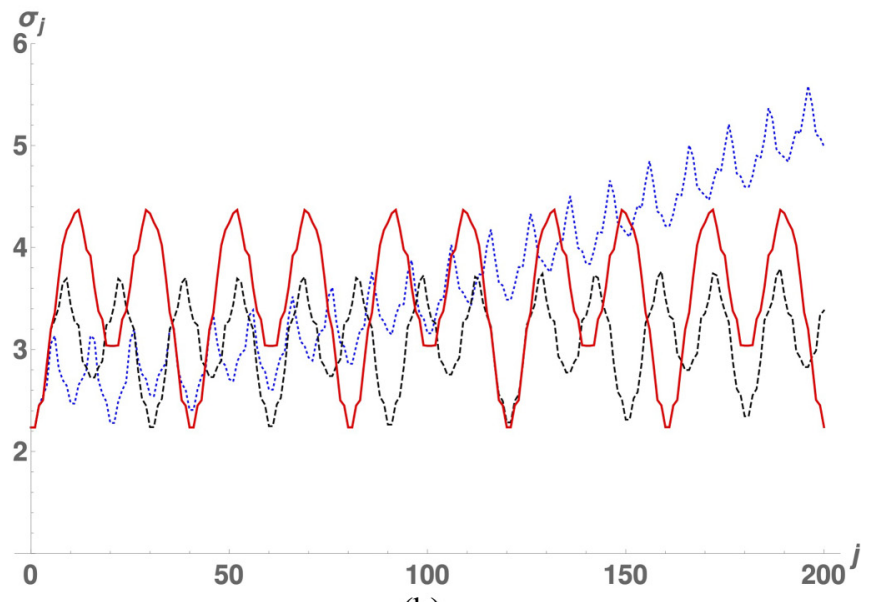

(b)

FIG. 4. (a) Average position $\langle n\rangle_{j}$ and (b) standard deviation $\sigma_{j}$ for initial Gaussian states [see Eq. (15)], as a function of the time instant $j$, for different field strengths: $\phi=2 \pi / 20$ (blue dotted line), $\phi=2 \pi / 30$ (black dashed line), and $\phi=2 \pi / 40$ (red solid line). The rest of the initial conditions are as in Fig. 3.

This two-step dynamics has already been considered in Ref. [29] (see also Ref. [30]) for the free DQW and in Ref. [5] for the electric DQW, although only in physical space and using the temporal gauge for the electric field [6]. Let us make use of the findings of these works: We assume that one can find two auxiliary partial states $A_{j}^{ \pm}(k)$ such that

$$
\tilde{\Psi}_{j}(k)=A_{j}^{+}(k)+(-1)^{j} A_{j}^{-}(k),
$$

with $A_{j}^{ \pm}(k)$ satisfying

$$
A_{j+1}^{ \pm}(k+\phi)-A_{j-1}^{ \pm}(k-\phi)= \pm \Delta_{\phi}(k) A_{j}^{ \pm}(k) .
$$

\section{B. Continuous-time approximation for weak electric fields and wide initial conditions}

\section{Introduction}

Without an electric field, it has been known since Strauch's work [25] that space-time-uniform DQWs are well approximated via space-time-uniform CQWs (which, if their graph is a lattice, are nothing but TBHs) if the DQW is chosen to be lazy, i.e., if the coin operator is almost a pure coin flip. 


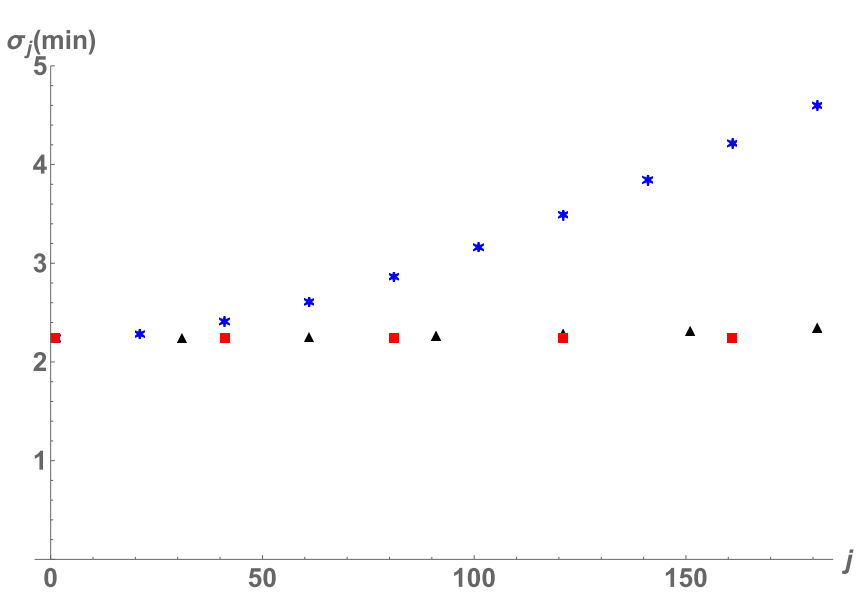

FIG. 5. The symbols represent the values of the standard deviation $\sigma_{j}$ corresponding to the minima in Fig. 4(b). Blue stars correspond to $\phi=2 \pi / 20$, black triangles to $\phi=2 \pi / 30$, and red squares to $\phi=2 \pi / 40$.

Before proceeding, let us give some details on the notion of the lazy DQW we are dealing with. When the coin operation is a pure coin flip, that is, the first Pauli matrix, $\sigma^{1}$, an initially localized walker, evolving via the free walk (3), does not move away from its original position in time. More precisely, it moves away by one lattice site when we apply the shift operation for the first time and then the right- and left-moving components are exchanged by the application of the pure coin flip operation so that the walker returns to its original position after applying the shift operation a second time; this backand-forth dynamics then goes "forever." A lazy DQW is when the coin operation is close to but not exactly a pure coin flip so that the walker does have a small probability of spreading away from its original position, controlled by the deviation to the pure coin flip operation, which is small. Instead of taking $\theta=\pi / 2$ in the coin operation defined in Eq. (9), which would yield a pure coin flip, one takes $\theta=\pi / 2-\delta$, with $\delta$ small with respect to $\pi / 2$ (for more details see Ref. [25] and Appendix A).

One can see with analytical arguments, or simply with numerical checks, that if the electric field of the electric DQW is chosen to be weak, then the above-mentioned continuoustime approximation will hold at least for some time and certain initial conditions and this time the Hamiltonian of the CQW will be an electric TBH, i.e., a standard TBH with an additional superimposed electric field. Notice that, from its definition (2), one can see a periodicity of $2 \pi$ in the variable $\phi$, which can therefore be restricted to the interval $[-\pi, \pi[$. According to this, a field will be called weak if it satisfies $|\phi| \ll \pi$. Now, as we have seen in Sec. II B, DQW Bloch oscillations actually hold even if the DQW is not chosen to be lazy, i.e., for arbitrary coin operators, and a continuoustime approximation is suggested by the smoothness of these oscillations when the initial condition is wide (see Fig. 3). In the free case (in the absence of electric field), one can show that a continuous-time approximation will hold at least for some time, even if the DQW is not chosen to be lazy, provided the initial condition is wide (details on this are given in Appendix A).
In what follows we assume that the continuous-time approximation holds for wide enough initial conditions, which will enable us to derive a formula that we will directly compare to numerical simulations of the original discrete-time dynamics.

We introduce a continuous-time variable $t$ and a time step $\tau>0$ and assume that $\tilde{\Psi}_{j}(k)$ coincides with a continuous function of time $\tilde{\Psi}(\cdot, k): t \mapsto \tilde{\Psi}(t, k)$ at instants $t_{j} \equiv j \tau$, i.e., $\tilde{\Psi}_{j}(k)=\tilde{\Psi}(t, k)$. We assume $\tilde{\Psi}(t, k)$ to be twice differentiable in $t$. Taylor expanding now the left-hand side of Eq. (23) at first order in both $\tau$ (continuous time) and $\phi$ (weak electric field), we arrive at

$$
i\left(\frac{\tau}{\phi} \partial_{t} A^{ \pm}(t, k)+\partial_{k} A^{ \pm}(t, k)\right)= \pm \frac{i}{2 \phi} \Delta_{\phi}(k) A^{ \pm}(t, k),
$$

which is a partial differential equation in both $t$ and $k$, where we have included the imaginary unit for convenience and the notation in the assumed continuous-time approximation should be clear from the context.

\section{Characteristic curve and two-step Hamiltonian}

The left-hand side of Eq. (24) possesses the characteristic curve $k_{t} \equiv k-\phi t / \tau$. Indeed, by introducing the new functions

$$
\breve{A}^{ \pm}\left(k, k_{t}\right) \equiv A^{ \pm}(t, k)
$$

we obtain

$$
i \partial_{k} \breve{A}^{ \pm}\left(k, k_{t}\right)= \pm \frac{i}{2 \phi} \Delta_{\phi}(k) \breve{A}^{ \pm}\left(k, k_{t}\right),
$$

which is an ordinary differential equation in $k$, i.e., time has been removed from the original equation (24). Let us examine Eq. (26) in more detail. It takes the form of a Schrödinger equation in which $k$ plays the role of time, up to the fact that $\Delta_{\phi}(k)$ [see Eq. (20)] is not Hermitian. Let us show that this lack of Hermiticity is actually only due to the fact that we have not yet performed the small-field approximation on $\Delta_{\phi}(k)$, which is needed for the consistency of the expansion. If we expand $\Delta_{\phi}(k)$ in $\phi$ and only keep the lowest contribution in $\phi$, Eq. (26) becomes

$$
i \partial_{k} \breve{A}^{ \pm}\left(k, k_{t}\right)= \pm \frac{1}{\phi} H_{2}(k) \breve{A}^{ \pm}\left(k, k_{t}\right),
$$

where we have introduced an operator that we call the twostep Hamiltonian

$$
H_{2}(k) \equiv \frac{i}{2}\left[W_{0}(k)-W_{0}^{\dagger}(k)\right],
$$

which is Hermitian.

This two-step Hamiltonian differs from the so-called effective Hamiltonian $H_{1}(k)$ that can be defined from $W_{0}(k)$ via

$$
W_{0}(k) \equiv e^{-i \tau H_{1}(k)} \text {. }
$$

We show in Appendix B that $H_{1}(k)$ and $H_{2}(k)$ are actually proportional,

$$
H_{1}(k)=\frac{\omega(k)}{\tau \sin \omega(k)} H_{2}(k),
$$

where $e^{ \pm i \omega(k)}$ are the eigenvalues of the matrix $W_{0}(k)$, assumed in $\mathrm{SU}(2)$. Equation (30) is a useful result for the free DQW, 
since the direct calculation of $H_{1}(k)$ via Eq. (29) is quite involved (see, e.g., Mallick's work [31]), while the calculation of $\mathrm{H}_{2}(k)$ defined by Eq. (28) is a trivial task.

\section{Explicit solutions in quasimomentum space for an arbitrary coin operator}

The solution of Eq. (27) can be written formally as

$$
A^{ \pm}(t, k) \equiv \breve{A}^{ \pm}\left(k, k_{t}\right)=V_{\phi}^{ \pm}(k) S^{ \pm}\left(k_{t}\right),
$$

where $S^{ \pm}(k)$ is a state that can be obtained from the initial conditions (discussed below) and we have defined the unitary operators

$$
V_{\phi}^{ \pm}(k)=\mathcal{T} \exp \left[\mp \frac{i}{\phi} \int_{0}^{k} d p H_{2}(p)\right] .
$$

We have to introduce the $p$-ordering $\mathcal{T}$ because in general $H_{2}(p)$ and $H_{2}\left(p^{\prime}\right)$ do not commute for $p \neq p^{\prime}$. Equation (32) determines, together with the knowledge of $A^{ \pm}(0, k)$, the state $S^{ \pm}(k)$ as

$$
S^{ \pm}(k) \equiv\left[V_{\phi}^{ \pm}(k)\right]^{\dagger} A^{ \pm}(0, k) .
$$

Putting everything together, i.e., plugging Eq. (33) into Eq. (31) and the resulting expression in Eq. (22), we arrive at

$$
\tilde{\Psi}(t, k)=\sum_{\alpha=+,-} \alpha^{t / \tau} V_{\phi}^{\alpha}(k)\left[V_{\phi}^{\alpha}\left(k_{t}\right)\right]^{\dagger} A^{\alpha}\left(0, k_{t}\right) .
$$

Let us now show that we can express the initial condition $A^{ \pm}(0, k)$ via $\tilde{\Psi}(0, k)$. Equation (22) taken for $j=0$ and $j=1$ yields, respectively,

$$
\begin{aligned}
\tilde{\Psi}(0, k) & =A^{+}(0, k)+A^{-}(0, k), \\
\tilde{\Psi}(\tau, k) & =A^{+}(\tau, k)-A^{-}(\tau, k) \\
& =A^{+}(0, k)-A^{-}(0, k)+O(\tau),
\end{aligned}
$$

so dropping the $O(\tau)$, i.e., at lowest order in $\tau$ (continuous-time approximation) and recalling that $\tilde{\Psi}(\tau, k)=W_{0}(k) \tilde{\Psi}(0, k)$ [equivalence condition between the one-step dynamics and the two-step one; see the discussion around Eq. (21)], we obtain

$$
A^{ \pm}(0, k)=\frac{1}{2}\left[1 \pm W_{0}(k)\right] \tilde{\Psi}(0, k) .
$$

Recall that if we insist on the possibility of having arbitrary angles $\theta$, the continuous-time approximation only holds in the long-wavelength approximation and this comes from the structure of mere free walk, as explained in Appendix A and as already noted in Ref. [29]. Choosing, furthermore, a weak electric field is necessary for this approximation to hold in the present case.

\section{Choice of a wide (spatially extended) initial condition and associated simplifications}

In what follows we consider a product initial state with the external-degree-of-freedom part defined in Eq. (14). In quasimomentum space it translates into

$$
\tilde{\Psi}(0, k)=g(k)|s\rangle,
$$

where

$$
g(k)=\sum_{n} c_{n} e^{-i k n}=\sqrt{\frac{\pi}{\beta}} e^{-k^{2} / 4 \beta} \frac{\vartheta_{3}\left(\frac{i k \pi}{2 \beta}, e^{-\pi^{2} / \beta}\right)}{\vartheta_{3}\left(0, e^{-2 \beta}\right)}
$$

is a quasimomentum amplitude distribution, which verifies $\int_{-\pi}^{\pi} \frac{d k}{2 \pi}|g(k)|^{2}=1$.

Assuming $g(k)$ peaked around $k=0$, i.e., the initial distribution to be spatially extended, allows for a further simplification in Eq. (38), namely,

$$
A^{ \pm}(0, k)=g(k) \Lambda^{ \pm}|s\rangle,
$$

where

$$
\Lambda^{ \pm} \equiv \frac{1}{2}\left[1 \pm W_{0}(0)\right]=\frac{1}{2}[1 \pm C]
$$

are the projectors on the eigenspaces of $W_{0}(0)=C$ and thus as projectors verify $\Lambda^{+} \Lambda^{+}=\Lambda^{+}, \Lambda^{-} \Lambda^{-}=\Lambda^{-}, \Lambda^{+} \Lambda^{-}=$ $\Lambda^{-} \Lambda^{+}=0$, and $\Lambda^{+}+\Lambda^{-}=1$. Using the above expressions, we can recast Eq. (34) as

$$
\tilde{\Psi}(t, k)=U(t, k) \tilde{\Psi}\left(0, k_{t}\right),
$$

having defined

$$
U(t, k) \equiv \sum_{\alpha=+,-} \alpha^{t / \tau} V_{\phi}^{\alpha}(k)\left[V_{\phi}^{\alpha}\left(k_{t}\right)\right]^{\dagger} \Lambda^{\alpha} .
$$

We can easily check that $U(t, k)$ is a unitary operator, and this holds because the $\Lambda^{ \pm}$are projectors, which is guaranteed if the initial condition is wide.

\section{Connection with a certain tight-binding Hamiltonian}

With our choice for the coin operator (9), we obtain

$$
H_{2}(k)=\cos \theta \sin k \mathbf{1}_{2},
$$

with $\mathbf{1}_{2}$ the $2 \times 2$ identity matrix. This result allows us to establish a remarkably simple connection with TBHs.

Indeed, consider the lattice Hamiltonian

$$
H(\hat{k}) \equiv i J\left(\hat{T}-\hat{T}^{\dagger}\right),
$$

where $\hat{T} \equiv e^{-i \hat{k}}=\sum_{n}|n+1\rangle\langle n|$ is the hopping operator to the right and $J \in \mathbb{R}$ is a constant, and recall that we have taken the lattice spacing $a=1$. In quasimomentum space, we obtain

$$
H(k)=2 J \sin k,
$$

which corresponds to the factor multiplying $\mathbf{1}_{2}$ in Eq. (45) provided we make the correspondence

$$
\cos \theta \leftrightarrow 2 J
$$

\section{Explicit solutions in quasimomentum space}

Since $\mathrm{H}_{2}(k)$ in Eq. (45) is proportional to $\mathbf{1}_{2}$, we can omit this trivial factor in what follows and then immediately get

$$
V_{\phi}^{ \pm}(k)=\exp \left[\mp \frac{i}{\phi} f(k)\right]
$$

where

$$
f(k) \equiv \cos \theta(1-\cos k)
$$

We also have

$$
\Lambda^{ \pm}=\left(\begin{array}{cc}
\cos ^{2} \frac{\theta}{2} & \pm \frac{1}{2} \sin \theta \\
\pm \frac{1}{2} \sin \theta & \sin ^{2} \frac{\theta}{2}
\end{array}\right)
$$


Making use of Eqs. (44), (39), and (49), we can finally write

$$
\tilde{\Psi}(t, k)=\left[F^{+}(t, k) \Lambda^{+}+(-1)^{t} F^{-}(t, k) \Lambda^{-}\right]|s\rangle,
$$

where

$$
F^{ \pm}(t, k) \equiv g\left(k_{t}\right) e^{\mp(i / \phi)\left[f(k)-f\left(k_{t}\right)\right]}
$$

and we have taken $\tau=1$ as the unit time step. Using some trigonometric identities, we can write

$$
f(k)-f\left(k_{t}\right)=-2 \cos (\theta) \sin (k-\phi t / 2) \sin (\phi t / 2) .
$$

\section{Explicit solutions in position space}

We know the relationship

$$
\Psi_{n}(t) \equiv\left\langle x_{n} \mid \Psi(t)\right\rangle=\frac{1}{2 \pi} \int_{-\pi}^{\pi} d k e^{i k n} \tilde{\Psi}(t, k),
$$

so taking the inverse Fourier transform of Eq. (52) yields

$$
\Psi_{n}(t)=\left[F_{n}^{+}(t) \Lambda^{+}+(-1)^{t} F_{n}^{-}(t) \Lambda^{-}\right]|s\rangle,
$$

where

$$
F_{n}^{ \pm}(t) \equiv \frac{1}{2 \pi} \int_{-\pi}^{\pi} d k e^{i k n} F^{ \pm}(t, k)
$$

Making use of Eq. (40), after some tedious but straightforward algebra we arrive at the final expressions

$$
\begin{array}{r}
F_{n}^{+}(t)=\sum_{l} c_{l} e^{i(n+l) \phi t / 2} J_{n-l}\left(2 \gamma \sin \frac{\phi t}{2}\right), \\
F_{n}^{-}(t)=\sum_{l} c_{l} e^{i(n+l) \phi t / 2} J_{-(n-l)}\left(2 \gamma \sin \frac{\phi t}{2}\right),
\end{array}
$$

with $c_{l}$ the initial coefficient of the wave function [see Eq. (14)], having introduced

$$
\gamma \equiv \frac{\cos \theta}{\phi}
$$

and where we have made use of the property $(-1)^{p} J_{p}(X)=$ $J_{-p}(X)$ holding for $p \in \mathbb{Z}$, the $J_{p}$ being Bessel functions of the first kind [32]. Notice that these expressions with Bessel functions are exactly those that appear in TBH models, if we make the correspondence $\cos \theta \leftrightarrow 2 J$. More precisely, the probability density reads

$$
\begin{aligned}
P_{n}(t) & \equiv \Psi_{n}^{\dagger}(t) \Psi_{n}(t) \\
& =\left|F_{n}^{+}(t)\right|^{2}\left\langle s\left|\Lambda^{+}\right| s\right\rangle+\left|F_{n}^{-}(t)\right|^{2}\left\langle s\left|\Lambda^{-}\right| s\right\rangle,
\end{aligned}
$$

so that one clearly sees that the solution is simply the probability sum of two counterpropagating solutions of the previously mentioned TBH [see Eq. (18) in Ref. [21]], weighted by the probabilities of going in one or the other direction given the initial coin state $|s\rangle$. Note that the fact that there are no interference terms between the two branches in the probability density is because the $\Lambda^{ \pm}$are projectors.

\section{Comparison between the $D Q W$ and its continuous-time approximation}

Figure 6 is a contour plot obtained with the same conditions as in Fig. 3, but using the approximated analytical result (56). As can be immediately seen, both plots agree visually very well.

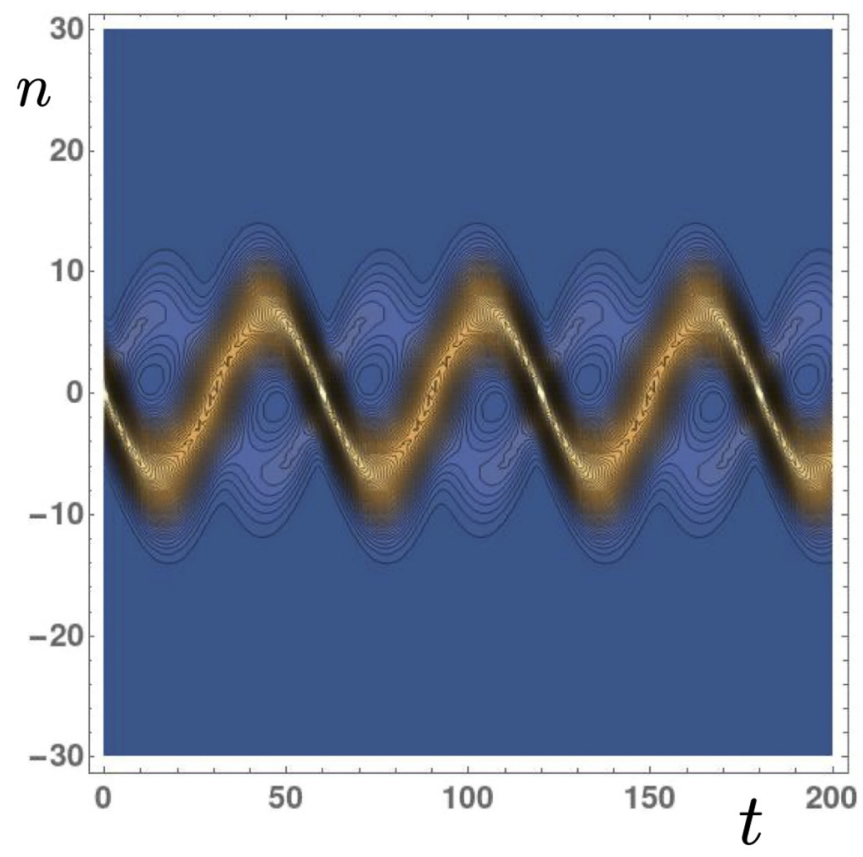

FIG. 6. Contour plots corresponding to the probability density $P_{n}(t)$, as a function of the lattice site $n$ and the time instant $t$, as obtained from Eq. (56). The initial conditions are the same as in Fig. 3(b).

In order to evaluate with more accuracy the agreement observed in comparing both contour plots, we show in Fig. 7 three different snapshots of the probability $P_{n}(t)$, as obtained from our approximated expression (56), in comparison with the exact numerical simulation. As can be appreciated from this figure, the degree of agreement can be different, depending on the exact time step, although it is generally good.

This figure also shows that the initial Gaussian exhibits a second small peak. The presence of this secondary peak arises from the combination of the two terms in Eq. (56) and is also visible in both Figs. 3 and 6. In fact, by choosing the initial coin state $|s\rangle$ to be one of the eigenstates of the coin operator $C$, then either $\Lambda^{+}|s\rangle=0$ or $\Lambda^{-}|s\rangle=0$, so the probability would not show such a secondary peak.

Another important result that can be obtained from the above approximated expressions is an analytical formula for both $\langle n\rangle_{t}$ and $\left\langle n^{2}\right\rangle_{t}$. Both calculations are performed in Appendix $\mathrm{C}$ and show an oscillatory result, with a period dictated by $T_{\mathrm{Bloch}}(\phi)$. As a consequence, our formulas predict that the DQW remains localized for any weak enough value of $\phi$ and a wide initial condition.

We would like to conclude this section with an important observation. We note that some expressions like the operator $V_{\phi}^{ \pm}(k)$ show a singular behavior at $\phi=0$ [see Eq. (49)]. Such a singular limit already appears in tight-binding models with an external electric field, where the Wannier-Stark eigenstates present this singularity [21]. However, observable magnitudes, for example, $\langle n\rangle_{t}$ or $\left\langle n^{2}\right\rangle_{t}$, do possess a well-defined limit as $\phi \rightarrow 0$, which can be obtained from Eqs. (C7) and (C10), giving

$$
\langle n\rangle_{t} \underset{\phi \rightarrow 0}{\longrightarrow} \kappa e^{-\beta / 2} t \sin \theta,
$$




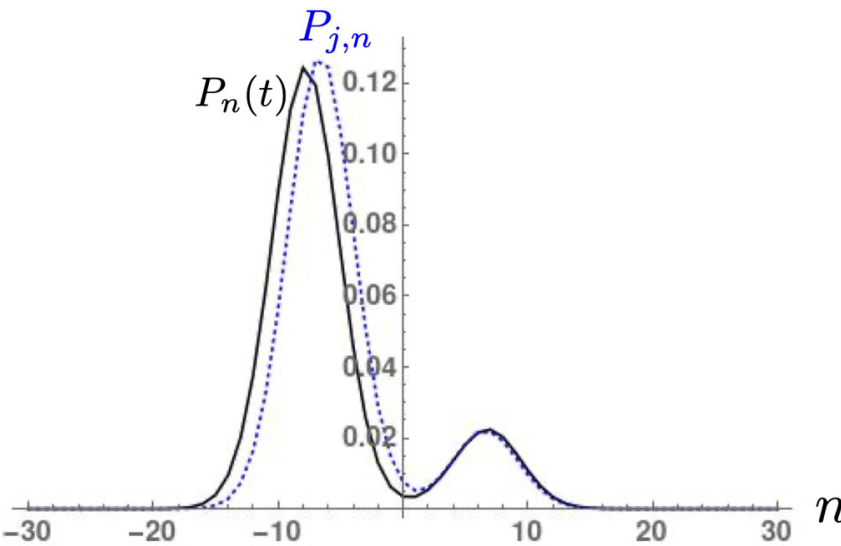

(a)

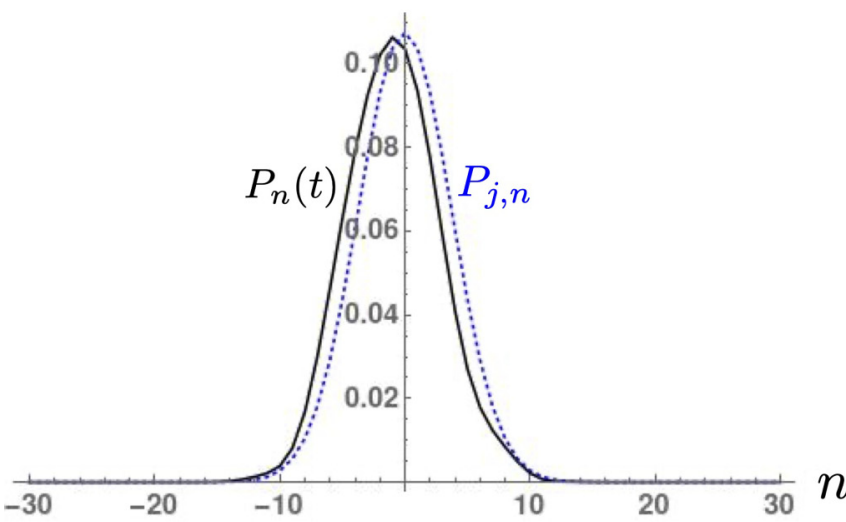

(b)

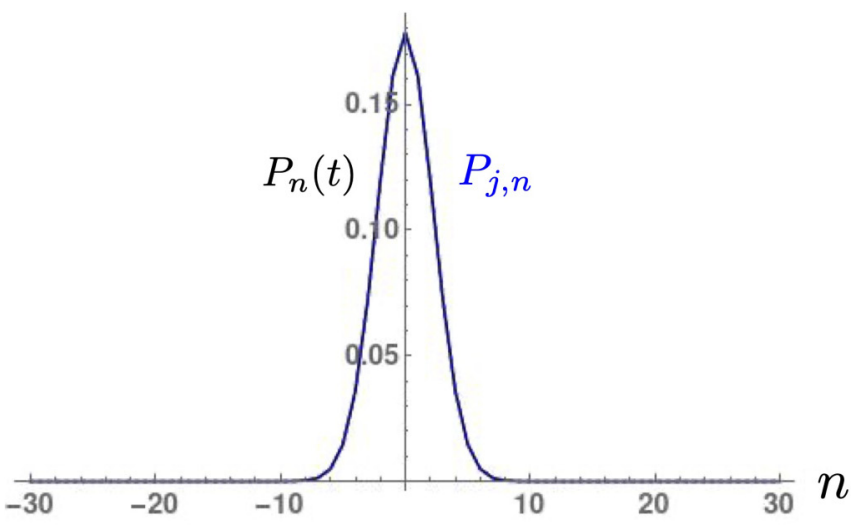

(c)

FIG. 7. Different snapshots of the probability $P_{n}(t)$, as obtained from Eq. (56) (black solid line), with the same parameters as in Fig. 6, in comparison with the exact simulation that gives $P_{j, n}$ (blue dotted line), for (a) $t=15$, (b) $t=30$, and (c) $t=60$. In this and similar plots, the points corresponding to different values of $n$ have been joined for better visualization. Notice that in the (c), $P_{j, n}$ and $P_{n}(t)$ are superposed.

which depends on the initial coin state via $\kappa$ defined in Eq. (C5), and

$$
\left\langle n^{2}\right\rangle_{t} \underset{\phi \rightarrow 0}{\longrightarrow} \frac{1}{4 \beta}+t^{2} \cos ^{2} \theta,
$$

which does not depend on the initial coin state. These two formulas are consistent with the ballistic propagation observed in the free DQW.

\section{MEASURE OF PROBABILITY AGREEMENT}

As we have observed in the preceding section, we have obtained good agreement between the calculated probability distribution $P_{n}(t)$ [see Eq. (61)], which comes from the approximated result of Eq. (56), and the exact simulation. In this section we would like to quantify this degree of agreement and, more importantly, to study how it changes with time. This question becomes very important if one wants to extract some conclusions about the long-term behavior of the DQW in the considered regime (weak electric field and wide initial condition).

In order to compare both probability distributions, we need some distance measure. There are in fact several measures available which are specially suited to compare two probability distributions, such as the Hellinger distance, the total variation distance, and the Kolmogorov-Smirnov distance (see [33] for a definition of these terms). All of them give extremely similar plots, so we concentrate on the Hellinger distance, which we write, for a given time instant $j$, as

$$
d_{j}^{\mathrm{H}}=\sqrt{1-\sum_{n} \sqrt{P_{j, n}^{\mathrm{ex}} P_{n}^{\mathrm{apr}}\left(t_{j}\right)},}
$$

where $P_{j, n}^{\text {ex }}$ is the exact probability distribution, whereas $P_{n}^{\text {apr }}\left(t_{j}\right)$ is obtained from Eq. (56) and evaluated at time $t_{j}=$ $j \tau$, with $\tau=1$. This distance will be bounded as $0 \leqslant d_{j}^{\mathrm{H}} \leqslant$ $1 \forall t$.

Figure 8(a) shows $d_{j}^{\mathrm{H}}$ for the same conditions used in previous figures. Several comments can be made about this figure. Within a numerical precision of the order $10^{-8}$, it is a periodic function of period $T_{\mathrm{Bloch}}(\phi)$, as expected from the results observed in the preceding section. It reaches a minimum value (numerically compatible with zero) at values of the time instant which are multiples of $T_{\mathrm{Bloch}}(\phi)$ [compare with Fig. 7(c)]. The maxima in $d_{j}^{\mathrm{H}}$ appear at time instants $j \simeq$ $T_{\text {Bloch }}(\phi) / 4$ [modulo $T_{\text {Bloch }}(\phi)$ ], with a constant value (considering the announced precision). It is important to mention that changing $\phi$ to an arbitrary close value does not change significantly the above plot, except for the fact that multiples or submultiples of $T_{\mathrm{Bloch}}(\phi)$ may not correspond to integer time instants $j$, a fact that introduces some small changes in the appearance of the figure. For this reason, we restrict ourselves to choices of $\phi$ giving an integer $T_{\mathrm{Bloch}}(\phi)$. Another important point to be made is that the above observations have been extracted from calculations which involve only a small number of Bloch periods. As discussed in Sec. II, as the value of $\phi$ is lowered, one quickly approaches a regime where the qualitative features of the probability distribution do not depend on $\phi$ and one has to consider much longer times (which would require a large amount of computational resources) to observe the differences.

We have explored different values of the field and of the initial conditions. Changing the intensity $\phi$ does not imply a significant modification of Fig. 8(a) (of course the period will be changed). A similar statement can be said about a 


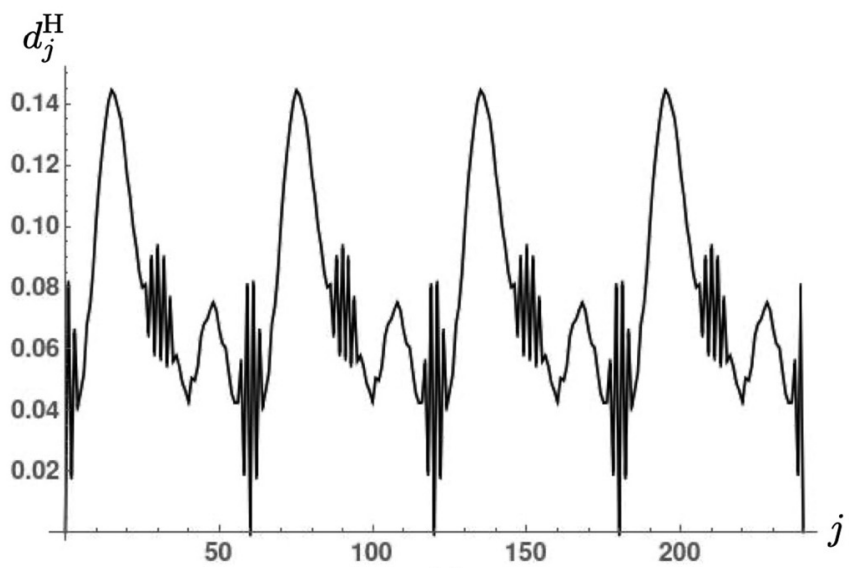

(a)

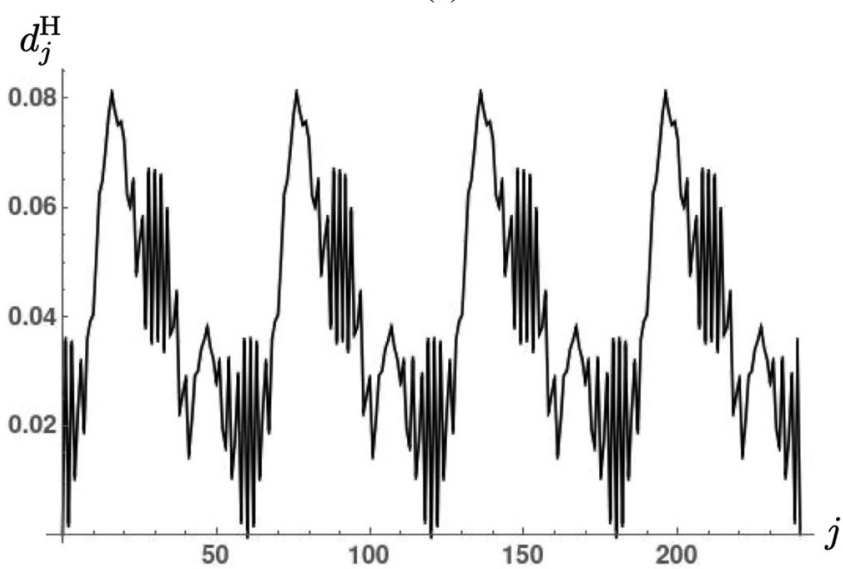

(b)

FIG. 8. Hellinger distance $d_{j}^{\mathrm{H}}$ between the exact and the approximated probability distributions, as a function of the time instant $j \in \mathbb{N}$. We have used the same parameters as in Fig. 6 in (a), i.e., in particular, $\beta=0.05$, and also for (b) except for the choice $\beta=0.01$.

modification of the initial coin state. Obviously, these statements only refer to the observed distance between both probability distributions, not to the overall evolution of the probability, which can be tailored by modifying the initial parameters, as discussed in Sec. III. The width of the initial Gaussian, however, has an important impact on the above figure. This can be appreciated in Fig. 8(b), which has been obtained with $\beta=0.01$, which implies a wider initial Gaussian. As can be seen, this introduces better agreement between the exact and the approximated distributions.

\section{CONCLUSION}

We presented a semianalytical study of the well-known electric DQW on the line [4] [Eq. (2)] in the case where the electric field $\phi$ is small $|\phi| \ll \pi$ and the initial condition is spatially extended. It was shown that, in such a regime, the dynamics of the electric DQW corresponds to semiclassical oscillations which are well approximated by an analytical continuous-time formula that is the sum of two counterpropagating solutions of a certain electric $\mathrm{TBH}$, that is, well-known semiclassical Bloch oscillations of a localized particle [21,22]. The hopping amplitude of this $\mathrm{TBH}$ is the cosine $\cos \theta$ of the arbitrary coin-operator mixing angle $\theta$. The result is semianalytical in the sense that the quality of the analytical approximation is evaluated numerically, via an appropriate distance measure. The price of the arbitrariness of $\theta$ is that the initial condition must be wide. If one wishes the continuous-time approximation to hold for spatially localized initial conditions, one needs at least the DQW to be lazy, as suggested by numerical simulations and by the fact that this has been analytically proven in the case of a vanishing electric field [25].

Finally, let us briefly mention how DQWs behave in the presence of noise. We first consider free, i.e., homogeneous, DQWs (in the context of this work, this means a vanishing electric field). These are generally not robust to noise, neither temporal nor spatial. Temporal noise on the coin-operation angle $\theta$ [see Eq. (9)] will generally cause diffusion to take over the typical ballistic motion of the DQW [34,35], while spatial noise on this coin-operation angle will generally cause Anderson localization [34]. Diffusive behavior also occurs when one performs, along the evolution, measurements on the coin or the spatial degree of freedom of the walker [36]. Considering electric DQWs, it was shown in Ref. [37] that a typical temporal disorder on the electric field also causes the walk to diffuse.

\section{ACKNOWLEDGMENTS}

We acknowledge illuminating discussions with Christopher Cedzich and Eugenio Roldán. This work was funded by the Spanish Ministerio de Economía, Industria y Competitividad, MINECO-FEDER Project No. FPA2017-84543-P, Grant No. SEV-2014-0398, and Generalitat Valenciana Grant No. PROMETEU/2019/087. We acknowledge support from CSIC Research Platform PTI-001.

\section{APPENDIX A: SIMPLE CONDITIONS TO APPROXIMATE A DQW BY A CQW}

We will derive the conditions for the free walk $W_{0}(\hat{k})$, defined in Eq. (3), which we write as $\mathcal{W}$ in the position representation, for the coin operator, defined in Eq. (9). We have, in the position representation,

$$
\begin{aligned}
& \Psi_{j+1, n}=\left(\mathcal{W} \Psi_{j}\right)_{n}, \\
& \Psi_{j-1, n}=\left(\mathcal{W}^{\dagger} \Psi_{j}\right)_{n},
\end{aligned}
$$

which yields

$$
\Psi_{j+1, p}-\Psi_{j-1, p}=\left[\mathcal{S}\left(\begin{array}{c}
c \psi_{j}^{R}+s \psi_{j}^{L} \\
s \psi_{j}^{R}-c \psi_{j}^{L}
\end{array}\right)\right]_{n}-C\left(\begin{array}{c}
\psi_{j, n+1}^{R} \\
\psi_{j, n-1}^{L}
\end{array}\right),
$$

with the coin components as defined in Eq. (5), where $\mathcal{S}$ is the position representation of $S(\hat{k})$ and we have used the notation

$$
\begin{aligned}
& \cos \theta=c, \\
& \sin \theta=s .
\end{aligned}
$$


Equation (A2) further results in

$\Psi_{j+1, n}-\Psi_{j-1, n}=\left(\begin{array}{l}c \psi_{j, n-1}^{R}+s \psi_{j, n-1}^{L}-c \psi_{j, n+1}^{R}-s \psi_{j, n-1}^{L} \\ s \psi_{j, n+1}^{R}-c \psi_{j, n+1}^{L}-s \psi_{j, n+1}^{R}+c \psi_{j, n-1}^{L}\end{array}\right)$,

which simplifies into the two (decoupled $\left.{ }^{2}\right)$ equations

$$
\begin{aligned}
& \psi_{j+1, n}^{R}-\psi_{j-1, n}^{R}=c\left(\psi_{j, n-1}^{R}-\psi_{j, n+1}^{R}\right), \\
& \psi_{j+1, n}^{L}-\psi_{j-1, n}^{L}=-c\left(\psi_{j, n+1}^{L}-\psi_{j, n-1}^{L}\right) .
\end{aligned}
$$

We want this discrete-time dynamics to be approximable by a continuous-time one, i.e., we want, for $u=L, R$,

$$
\left|\psi_{j+1, n}^{u}-\psi_{j-1, n}^{u}\right|=|c|\left|\psi_{j, n-1}^{u}-\psi_{j, n+1}^{u}\right| \ll\left|\psi_{j-1, n}^{u}\right| .
$$

As Eq. (A6) suggests, a first possibility for this to be satisfied at least for some time, even if the initial condition is not wide in space, is, as shown in Ref. [25] (we will not give additional information here, but refer the reader to that reference), to choose $c$ small enough, i.e., $C$ to be almost a pure coin flip. If one starts with a Dirac delta at $n=0$ initially, one ends up, after two steps, with almost the same Dirac delta at $n=0$, the amplitude being diminished by a small amount only, so that the continuous-time approximation is good. As Eq. (A6) suggests, a second possibility for the continuoustime approximation to hold at least for some time whatever angle $\theta$ we choose is to take an initial condition which is wide in space, as already considered for the free walk in Ref. [29] and for the electric walk in the temporal gauge in Ref. [5] (in the present work we chose the spatial gauge because it is simpler in quasimomentum space). Note that the quality of the continuous-time approximation depends on the joint effect of these two conditions; it is only in the two respective limits $(\theta \rightarrow \pi / 2$ or the infinite-wavelength limit) that one can forget about the other condition.

\section{APPENDIX B: RELATIONSHIP BETWEEN THE TWO-STEP HAMILTONIAN $H_{2}(K)$ AND THE EFFECTIVE HAMILTONIAN $H_{1}(k)$}

In this Appendix we prove Eq. (30), holding when the free walk $W_{0}(k)$ is special unitary, i.e., belonging to $\mathrm{SU}(2)$, so that its eigenvalues can be written $e^{ \pm i \omega(k)}$. Note that this condition of special unitarity is not satisfied with the coin operator of Eq. (9). We can write

$$
W_{0}(k)=\mathcal{U}(k)\left(\begin{array}{cc}
e^{-i \omega(k)} & 0 \\
0 & e^{i \omega(k)}
\end{array}\right) \mathcal{U}^{\dagger}(k),
$$

where $\mathcal{U}(k)$ is a unitary matrix containing the eigenvectors of $W_{0}(k)$.

Now, on the one hand, from the definition of $H_{1}(k)$ in Eq. (29), and assuming the principal branch for the logarithm, we arrive at

$$
H_{1}(k)=\frac{\omega(k)}{\tau} \mathcal{U}(k) \sigma_{z} \mathcal{U}^{\dagger}(k)
$$

\footnotetext{
${ }^{2}$ The decoupling arises here because the coin operator $C$ is Hermitian.
}

On the other hand, from Eq. (28) we easily obtain

$$
H_{2}(k)=\sin \omega(k) \mathcal{U}(k) \sigma_{z} \mathcal{U}^{\dagger}(k) .
$$

By combining both expressions, Eq. (30) immediately follows.

\section{APPENDIX C: AVERAGE POSITION AND STANDARD DEVIATION}

The average position defined in Eq. (12) can be more easily calculated in quasimomentum space as

$$
\langle n\rangle_{t}=\frac{1}{2 \pi} \int_{-\pi}^{\pi} d k \tilde{\Psi}^{\dagger}(t, k) i \partial_{k} \tilde{\Psi}(t, k),
$$

where $\tilde{\Psi}^{\dagger}(t, k)$ is obtained by transposition and complex conjugation from $\tilde{\Psi}(t, k)$. Using the expression of $\tilde{\Psi}(t, k)$ given by Eq. (52) and making use of the properties of the projectors $\Lambda^{ \pm}$, we can write

$$
\begin{aligned}
\langle n\rangle_{t}= & \frac{i}{2 \pi} \int_{-\pi}^{\pi} d k\left[F^{+*}(t, k) \partial_{k} F^{+}(t, k)\left\langle s\left|\Lambda^{+}\right| s\right\rangle\right. \\
& \left.+F^{-*}(t, k) \partial_{k} F^{-}(t, k)\left\langle s\left|\Lambda^{+}\right| s\right\rangle\right] .
\end{aligned}
$$

Writing the coin state as

$$
|s\rangle \equiv\left(\begin{array}{l}
a \\
b
\end{array}\right)
$$

with the condition that $|a|^{2}+|b|^{2}=1$, Eq. (C2) yields

$$
\begin{aligned}
\langle n\rangle_{t}= & \frac{1}{2 \pi \phi} \int_{-\pi}^{\pi} d k g\left(k_{t}\right)\left[\phi g^{\prime}\left(k_{t}\right)-2 i \kappa \cos (\theta) g\left(k_{t}\right)\right. \\
& \left.\times \sin \left(\frac{\phi t}{2}\right) \cos \left(k-\frac{\phi t}{2}\right)\right],
\end{aligned}
$$

where

$$
\kappa \equiv\langle s|C| s\rangle=b^{*}(a \sin \theta-b \cos \theta)+a^{*}(a \cos \theta+b \sin \theta)
$$

is the mean value of the coin operator $C$ in the initial state $|s\rangle$, which here is a real number because $C$ is Hermitian. The exact computation of this integral yields an impractical result because the expression of $g(k)$ [see Eq. (40)] is too complicated. However, since we only consider the long-wavelength limit, we can replace the summation in the definition of $g(k)$ by an integral, which amounts to regarding this function as the Fourier transform of the continuous function $c(n) \equiv c_{n}$, where $c_{n}$ is defined by Eq. (15). In this way, we approximate

$$
g(k) \simeq(2 \pi \beta)^{1 / 4} e^{-k^{2} / 4 \beta} .
$$

Since we are interested in low values of $\beta$, the integration limits in Eq. (C4) become $\pm \infty$. With these approximations, the above integral can be performed, resulting in

$$
\langle n\rangle_{t}=\kappa e^{-\beta / 2} \sin (\theta) \frac{\sin (\phi t)}{\phi} .
$$

A similar procedure can be followed to obtain $\left\langle n^{2}\right\rangle_{t}$. Similarly to Eq. (C2), we have

$$
\begin{aligned}
\left\langle n^{2}\right\rangle_{t}= & -\frac{1}{2 \pi} \int_{-\pi}^{\pi} d k\left[F^{+*}(t, k) \partial_{k}^{2} F^{+}(t, k)\left\langle s\left|\Lambda^{+}\right| s\right\rangle\right. \\
& \left.+F^{-*}(t, k) \partial_{k}^{2} F^{-}(t, k)\left\langle s\left|\Lambda^{-}\right| s\right\rangle\right] .
\end{aligned}
$$


By performing the same approximations as done in the calculation of $\langle n\rangle_{t}$, we finally obtain the expression

$$
\left\langle n^{2}\right\rangle_{t}=\frac{1}{4 \beta}+2 \cos ^{2}(\theta) \frac{\sin ^{2}\left(\frac{\phi t}{2}\right)}{\phi^{2}}\left[1+e^{-2 \beta} \cos (\phi t)\right]
$$

Using the fact that the initial Gaussian [see Eq. (15)] is wide, i.e., that $\beta$ is small, so that $e^{-2 \beta} \simeq 1$, the previous result (C9) can be further approximated by

$$
\left\langle n^{2}\right\rangle_{t} \simeq \frac{1}{4 \beta}+\cos ^{2}(\theta) \frac{\sin ^{2}(\phi t)}{\phi^{2}} .
$$

[1] A. Ambainis, A. M. Childs, B. W. Reichardt, R. Špalek, and S. Zhang, SIAM J. Comput. 39, 2513 (2010).

[2] G. Wang, Quantum Inf. Comput. 17, 987 (2017).

[3] P. Arnault, Ph.D. thesis, Université Pierre et Marie Curie, 2017.

[4] C. Cedzich, T. Rybár, A. H. Werner, A. Alberti, M. Genske, and R. F. Werner, Phys. Rev. Lett. 111, 160601 (2013).

[5] M. C. Bañuls, C. Navarrete, A. Pérez, E. Roldán, and J. C. Soriano, Phys. Rev. A 73, 062304 (2006).

[6] G. Di Molfetta, F. Debbasch, and M. Brachet, Physica A 397, 157 (2014).

[7] L. A. Bru, M. Hinarejos, F. Silva, G. J. de Valcárcel, and E. Roldán, Phys. Rev. A 93, 032333 (2016).

[8] M. Genske, W. Alt, A. Steffen, A. H. Werner, R. F. Werner, D. Meschede, and A. Alberti, Phys. Rev. Lett. 110, 190601 (2013).

[9] P. Arnault and F. Debbasch, Physica A 443, 179 (2015).

[10] P. Arnault and F. Debbasch, Phys. Rev. A 93, 052301 (2016).

[11] I. Yalçınkaya and Z. Gedik, Phys. Rev. A 92, 042324 (2015).

[12] P. Arnault and F. Debbasch, Ann. Phys. (NY) 383, 645 (2017).

[13] P. Arrighi and S. Facchini, Quantum Inf. Comput. 17, 810 (2017).

[14] P. Arrighi, G. Di Molfetta, I. Márquez-Martín, and A. Pérez, Sci. Rep. 9, 10904 (2019).

[15] G. Di Molfetta and A. Pérez, New J. Phys. 18, 103038 (2016).

[16] L. A. Bru, G. J. de Valcárcel, G. Di Molfetta, A. Pérez, E. Roldán, and F. Silva, Phys. Rev. A 94, 032328 (2016).

[17] A. Regensburger, C. Bersch, B. Hinrichs, G. Onishchukov, A. Schreiber, C. Silberhorn, and U. Peschel, Phys. Rev. Lett. 107, 233902 (2011).

[18] D. Witthaut, Phys. Rev. A 82, 033602 (2010).

[19] A. Alberti and S. Wimberger, Phys. Rev. A 96, 023620 (2017).

[20] V. V. Ramasesh, E. Flurin, M. Rudner, I. Siddiqi, and N. Y. Yao, Phys. Rev. Lett. 118, 130501 (2017).
[21] T. Hartmann, F. Keck, H. J. Korsch, and S. Mossmann, New J. Phys. 6, 2 (2004).

[22] F. Domínguez-Adame, Eur. J. Phys. 31, 639 (2010).

[23] D. Tamascelli, S. Olivares, S. Rossotti, R. Osellame, and M. G. A. Paris, Sci. Rep. 6, 26054 (2016).

[24] D. Witthaut, F. Keck, H. J. Korsch, and S. Mossmann, New J. Phys. 6, 41 (2004).

[25] F. W. Strauch, Phys. Rev. A 74, 030301(R) (2006).

[26] D. A. Meyer, J. Stat. Phys. 85, 551 (1996).

[27] C. Cedzich and A. H. Werner, arXiv:1906.11931.

[28] T. Geisel, R. Ketzmerick, and G. Petschel, Phys. Rev. Lett. 66, 1651 (1991).

[29] P. L. Knight, E. Roldán, and J. E. Sipe, Phys. Rev. A 68, 020301(R) (2003).

[30] A. Romanelli, A. C. S. Schifino, R. Siri, G. Abal, A. Auyuanet, and R. Donangelo, Physica A 338, 395 (2004).

[31] A. Mallick, Ph.D. thesis, The Institute of Mathematical Sciences of Chennai, 2019.

[32] I. S. Gradshteyn and I. M. Ryzhik, in Table of Integrals, Series, and Products, 8th ed., edited by D. Zwillinger (Academic Press, 2014).

[33] A. Dobson, Stat. Med. 23, 1824 (2004).

[34] A. Ahlbrecht, C. Cedzich, R. Matjeschk, V. B. Scholz, A. H. Werner, and R. F. Werner, Quantum Inf. Process. 11, 1219 (2012).

[35] P. Arnault, A. Macquet, A. Anglés-Castillo, I. MárquezMartín, V. Pina-Canelles, A. Pérez, G. D. Molfetta, P. Arrighi, and F. Debbasch, J. Phys. A: Math. Theor. 53, 205303 (2020).

[36] J. Kempe, Contemp. Phys. 44, 307 (2003).

[37] G. Di Molfetta and F. Debbasch, Quantum Stud. 3, 293 (2016) 